\title{
ALTERNATIVE FORMS OF FRACTIONAL BROWNIAN MOTION ${ }^{*}$
}

\author{
by \\ D Marinucci and P M Robinson \\ London School of Economics and Political Science
}

\section{Contents:}

Abstract

1. Introduction

2. "Type I" Fractional Brownian Motion

3. "Type II" Fractional Brownian Motion

4. Conclusion

References

$\begin{array}{ll} & \text { The Suntory Centre } \\ & \text { Suntory and Toyota International Centres } \\ \text { for Economics and Related Disciplines } \\ \text { London School of Economics and Political } \\ \text { Science } \\ \text { Houghton Street } \\ \text { Discussion Paper } & \text { London WC2A } 2 A E \\ \text { No.EM/98/354 } & \text { Tel.: } 0171-4057686 \\ \text { July } 1998 & \end{array}$

* Research supported by ESRC grant R000235892. We are grateful to two referees for comments that improved the presentation; the first author has also benefited from a useful discussion with P Baldi. 


\begin{abstract}
It is pointed out that two contradictory definitions of fractional Brownian motion are well established, one prevailing in the probabilistic literature, the other in the econometric literature. Each is associated with a different definition of nonstationary fractional time series, arising in functional limit theorems based on such series. These various definitions have occasionally led to some confusion. The paper discusses the definitions and attempts a clarification.
\end{abstract}

Keywords: Fractional Brownian motion; nonstationary time series; longrange dependence.

JEL No.: C32

(C) by D Marinucci and P M Robinson. All rights reserved. Short sections of text, not to exceed two paragraphs, may be quoted without explicit permission provided that full credit, including (c) notice, is given to the source. 


\section{INTRODUCTION}

We define (standard) Brownian motion $B(r), r \in R$, to be a real-valued Gaussian process with

$$
E B(r)=0, r \in R, \quad E B\left(r_{1}\right) B\left(r_{2}\right)=\min \left(r_{1}, r_{2}\right), r_{1}, r_{2} \geq 0 .
$$

$B(r)$ has independent increments, and indeed for integers $j=0, \pm 1, \ldots$, the sequence of increments

$$
b(j)=B(j+1)-B(j)
$$

are independent and identically distributed (i.i.d.) standard Gaussian variates. The sample paths of $B(r)$ are almost all continuous, $B(r)$ is $\frac{1}{2}-s s$ (self-similar with index $\frac{1}{2}$ ); a process, $X(r), r \in R$, is said to be $H-s s$ if the finite-dimensional joint distributions of $X(a r)$ are identical to those of $a^{H} X(r)$, for all $a>0$.

Functional central limit theorems (weak invariance principles) entail weak convergence of random variables to Brownian motion in a suitable metric space. A good introduction to this topic is given by Heyde (1981) with a particularly detailed treatment by Billingsley (1968). Denote by $(X, \delta)$ a complete separable metric space with metric $\delta$ and probability measures $\mu_{i}, i \geq 0$, on the Borel sets of $X$. We say that $\mu_{n}$ converges weakly to $\mu_{0}$ in $(X, \delta)$ if for every bounded continuous function $f$ in $X, \lim _{n \rightarrow \infty} \int f d \mu_{n}=\int f d \mu_{0}$. We can construct a probability space $\{\Omega, \Im, P\}$ with random elements $\xi_{n}, n \geq 0$, of $X$ having distributions $\mu_{n}$ respectively. If $\mu_{n}$ converges weakly to $\mu_{0}$ then we write $\xi_{n} \Rightarrow \xi_{0}$. Two metric spaces which feature considerably in the theory of weak convergence are $C$, the space of continuous functions on $[0,1]$ endowed with the uniform topology, and $D$, the space of functions on $[0,1]$ that are continuous on the right with finite left limits, endowed with the Skorohod (1956) $J_{1}$ topology.

Consider partial sums of a sequence $u_{t}, t=1,2, \ldots$ of random variables. Let

$$
S_{0}=0
$$




$$
S_{n}=u_{1}+\ldots+u_{n}, \quad n \geq 1,
$$

and define the polygonal line function

$$
S_{n}(r)=S_{[n r]}+(n r-[n r]) u_{[n r]+1}, \quad 0 \leq r \leq 1 .
$$

Notice that $S_{n}(r) \in C$ and $S_{[n r]} \in D$. In case the $u_{t}$ are independent and identically distributed (i.i.d.) with zero mean and unit variance then Donsker's theorem (see Donsker (1951), Prohorov (1956), Skorohod (1956)) asserts that

$$
n^{-\frac{1}{2}} x_{n}(r) \Rightarrow B(r), \quad \text { as } \quad n \rightarrow \infty, \quad 0 \leq r \leq 1,
$$

for $x_{n}(r)=S_{[n r]}$ or $S_{n}(r)$. Clearly $x_{n}(r)$ can be centered and scaled to cope with alternative values of $E u_{t}$ and $\operatorname{Var}\left(u_{t}\right)$. The proof of (1.6) on both $C$ and $D$ entails establishing convergence of finite-dimensional distributions and tightness, for which a sufficient condition (see Billingsley (1968), p.128) is that for some $\gamma>0, \alpha>1$

$$
E\left\{\left|x_{n}(t)-x_{n}\left(t_{1}\right)\right|^{\gamma}\left|x_{n}\left(t_{2}\right)-x_{n}(t)\right|^{\gamma}\right\} \leq K\left|t_{2}-t_{1}\right|^{\alpha},
$$

for some $K<\infty$ and all $t, t_{1}, t_{2}$ such that $0 \leq t_{1} \leq t \leq t_{2} \leq 1$.

The convergence (1.6) has been extended to many classes of dependent random variables. Brown (1971) and subsequent authors considered martingale difference $u_{t}$. Much literature has allowed for autocorrelation in $u_{t}$. Suppose now that $u_{t}$ is covariance stationary and has (without loss of generality) mean zero, and lag- $j$ autocovariance $\gamma(j)=E u_{t} u_{t+j}$. Define

$$
f(0)=\frac{1}{2 \pi} \sum_{j=-\infty}^{\infty} \gamma(j)
$$

assuming that

$$
0<f(0)<\infty
$$

Under a variety of conditions such that (1.7) holds, for example with $u_{t}$ a linear process (e.g. Hannan (1979), Phillips and Solo (1992)), various mixing or functions-of-mixing processes (e.g. McLeish (1977), Herrndorf (1984), Wooldridge and White (1988)), or with vector 
valued $u_{t}$ (e.g. Phillips and Durlauf (1986)), we have

$$
\{2 \pi f(0) n\}^{-\frac{1}{2}} x_{n}(r) \Rightarrow B(r), \quad \text { as } n \rightarrow \infty, \quad 0 \leq r \leq 1 .
$$

A leading motivation for much of this work has been its application to limit distribution theory for statistics that arise when investigating the possibility that an observed time series has a unit root, against the alternative that it has autoregressive stationarity or explosivity; often application of functional limit theorems of the form (1.6) or (1.8), and the continuous mapping theorem (see Billingsley (1968)), leads to limit distributions that are nonstandard functionals of Brownian motion.

In case $u_{t}$ has absolutely continuous spectral distribution, $f(0)$ is the ordinate of the spectral density function, $f(\lambda)$, at $\lambda=0$. The property (1.7) can be viewed as a mild form of short-range dependence condition (while it is also possible to focus on behaviour at alternative frequencies $\lambda$ ). Some of the work establishing (1.8) has allowed for forms of nonstationarity requiring $f(0)$ to have a broader interpretation, but nevertheless (1.7) still conveys a sense of weak dependence. While many standard time series models for $u_{t}$, including stationary and invertible mixed autoregressive moving averages, satisfy (1.7), there has been considerable interest in ones which do not, and these lead to an interest in forms of fractional Brownian motion.

\section{2. "TYPE I" FRACTIONAL BROWNIAN MOTION}

Mandelbrot and Van Ness (1968) introduced fractional Brownian motion $B_{H}(r)$ which we present in the slightly modified form of Samorodnitsky and Taqqu (1994) (see also Taqqu (1979)), for $0<H<1$ :

$$
B_{H}(r)=\frac{1}{A(H)} \int_{R}\left[\left\{(r-s)_{+}\right\}^{H-\frac{1}{2}}-\left\{(-s)_{+}\right\}^{H-\frac{1}{2}}\right] d B(s), \quad r \in R,
$$

where $(t)_{+}=\max (t, 0)$ and

$$
A(H)=\left\{\frac{1}{2 H}+D(H)\right\}^{\frac{1}{2}}, \quad D(H)=\int_{0}^{\infty}\left\{(1+s)^{H-\frac{1}{2}}-s^{H-\frac{1}{2}}\right\}^{2} d s .
$$


We term $B_{H}(r)$ "Type I" fractional Brownian motion.

For $H=\frac{1}{2}(2.1)$ is interpreted as

$$
\begin{gathered}
B_{H}(r)=\int_{0}^{r} d B(s), \quad r \geq 0, \\
B_{H}(r)=-\int_{r}^{0} d B(s), \quad r<0,
\end{gathered}
$$

so that (1.1) is satisfied. For $H \neq \frac{1}{2}$ can be formally interpreted as a fractional derivative or integral of $B(r)$ in the sense of Weyl (1917) (see Zygmund (1977), chapter XII). We can rewrite $(2.1)$ as

$$
\begin{gathered}
B_{H}(r)=\frac{1}{A(H)} \int_{-\infty}^{0}\left\{(r-s)^{H-\frac{1}{2}}-(-s)^{H-\frac{1}{2}}\right\} d B(s) \\
+\frac{1}{A(H)} \int_{0}^{r}(r-s)^{H-\frac{1}{2}} d B(s)
\end{gathered}
$$

for $r \geq 0$ and

$$
\begin{gathered}
B_{H}(r)=\frac{1}{A(H)} \int_{-\infty}^{r}\left\{(r-s)^{H-\frac{1}{2}}-(-s)^{H-\frac{1}{2}}\right\} d B(s)+ \\
-\frac{1}{A(H)} \int_{r}^{0}(-s)^{H-\frac{1}{2}} d B(s)
\end{gathered}
$$

for $r<0$. It is then easily verified that $E B_{H}(r)=0, r \in R$, and

$$
E B_{H}\left(r_{1}\right) B_{H}\left(r_{2}\right)=\frac{1}{2}\left(\left|r_{1}\right|^{2 H}+\left|r_{2}\right|^{2 H}-\left|r_{2}-r_{1}\right|^{2 H}\right), \quad r_{1}, r_{2} \in R .
$$

It follows that the increment $B_{H}\left(r_{2}\right)-B_{H}\left(r_{1}\right), r_{2} \geq r_{1}$, has variance

$$
E\left(B_{H}\left(r_{2}\right)-B_{H}\left(r_{1}\right)\right)^{2}=\left|r_{2}-r_{1}\right|^{2 H} .
$$

Thus for integers $j=0, \pm 1, \ldots$ the increments

$$
b_{H}(j)=B_{H}(j+1)-B_{H}(j)
$$

have zero mean, unit variance and autocovariance

$$
\operatorname{Cov}\left(b_{H}(j), b_{H}(k)\right)=\frac{1}{2}\left(|j-k+1|^{2 H}-2|j-k|^{2 H}+|j-k-1|^{2 H}\right) .
$$


Hence they have standard Gaussian marginals and are stationary like (1.2) but, unlike (1.2), are autocorrelated when $H \neq \frac{1}{2}$, so, writing $(2.9)$ as $\rho_{H}(j-k)$ we have

$$
\rho_{H}(j) \sim H(2 H-1) j^{2 H-2}, \quad \text { as } j \rightarrow \infty
$$

where " $"$ means that the ratio of left- and right-hand side tends to one. Mandelbrot and Van Ness (1968) showed that $B_{H}(r)$ has almost all sample paths continuous, and is $H-s s$. Verwaat (1985) showed that $B_{H}(r)$ can only be defined for $H>0$ in that for $H \leq 0$ an $H-s s$ process with stationary increments is almost surely identically zero, and also showed that for $0<H<1$ the paths of $B_{H}(r)$ have almost surely locally unbounded variations, in common with standard Brownian motion $B(r)$. Samorodnitsky and Taqqu (1994) indicate that $B_{H}(r)$ is not a unique representation of fractional Brownian motion, in that for any real $a$ and $b$ the process

$$
\int_{R}\left[a\left\{(r-s)_{+}^{H-\frac{1}{2}}-(-s)_{+}^{H-\frac{1}{2}}\right\}+b\left\{(r-s)_{-}^{H-\frac{1}{2}}-(-s)_{-}^{H-\frac{1}{2}}\right\}\right] d B(s), \quad r \in R
$$

shares the same properties as $B_{H}(r)$, up to a multiplicative constant, where $(t)_{-}=-\min (t, 0)$; (2.11) provides a general expression for "moving average" representations of fractional Brownian motion. Samorodnitsky and Taqqu (1994) also discuss "harmonizable representations", which for real scalar $a$ and $b$ they present as

$$
\int_{-\infty}^{\infty} \frac{e^{i \lambda r}-1}{i \lambda}\left(a(\lambda)_{+}^{-(H-1 / 2)}+b(\lambda)_{-}^{-(H-1 / 2)}\right) d M(\lambda), r \in R
$$

where $d M(\lambda)$ is a complex Gaussian random measure, such that

$$
d M(\lambda)=d \overline{M(-\lambda)}, E d M(\lambda)=0, E|d M(\lambda)|^{2}=d \lambda
$$

the bar denoting complex conjugation, and

$$
\operatorname{EdM}(\lambda)^{2}=E d M(\lambda) d \overline{M(\mu)}=0, \lambda \neq \mu
$$

The representation (2.12) was introduced by Dobrushin (1979) and Dobrushin and Major (1979), while its equivalence (in the finite-dimensional distribution sense) with (2.11) was first proved by Taqqu (1979). 
We now consider how $B_{H}(r)$ describes the limiting behaviour of partial sums of certain random variables $u_{t}$. The increment sequence $b_{H}(j)(2.8)$ provides a clue as to the properties of $u_{t}$. Corresponding to $(2.6)$, the stationary sequence $b_{H}(j)$ has spectral density $h(\lambda)$, $-\pi<\lambda \leq \pi$, satisfying

$$
h(\lambda) \sim\left\{\frac{H \Gamma(2 H) \sin \pi H}{\pi}\right\} \lambda^{1-2 H}, \quad \text { as } \lambda \rightarrow 0^{+} .
$$

Thus it is seen that

$$
\begin{aligned}
& h(0)=\infty, \quad \frac{1}{2}<H<1, \\
& h(0)=0, \quad 0<H<\frac{1}{2},
\end{aligned}
$$

so by comparison with $(1.7)$ the $b_{H}(j)$ are not "short-range dependent". Correspondingly, the $u_{t}$ whose partial sums are approximated by $B_{H}(r)$ typically have autocovariances $\gamma(j)$ that, up to a multiplicative constant, are approximated by the right side of (2.10), and/or have spectral density $f(\lambda)$ that is approximated, up to a multiplicative constant, by the right side of (2.13). In view of the factor $f(0)$ in (1.8) the behaviour of $f(\lambda)$ near $\lambda=0$ is of interest in the present context; considering the class $f(\lambda)$ satisfying

$$
f(\lambda) \sim C \lambda^{1-2 H}, \quad \text { as } \lambda \rightarrow 0^{+}, \quad 0<C<\infty,
$$

we say that $u_{t}$ is long-range dependent under $\frac{1}{2}<H<1$ (so $f(0)=\infty$ ) and negative dependent when $0<H<\frac{1}{2}$ (so $f(0)=0$ ), in both of which cases (1.7) is invalidated.

Assuming that the closely related condition to (2.14) (see Yong, 1974)

$$
\gamma(j) \sim c H(2 H-1) j^{2 H-2}, \quad \text { as } j \rightarrow \infty
$$

for $0<H<\frac{1}{2}$ or $\frac{1}{2}<H<1$ and for $0<c<\infty$ (cf. (2.10)) it follows that $V\left(S_{n}\right) \sim c n^{2 H}$ as $n \rightarrow \infty$. Thus we anticipate that under (2.15) and regularity conditions

$$
c^{-\frac{1}{2}} n^{-H} x_{n}(r) \Rightarrow B_{H}(r), \quad 0<r \leq 1
$$

for $x_{n}(r)=S_{n}(r)$ or $S_{[n r]}$. Davydov (1970) established (2.16) in the former case when $u_{t}$ is a linear process with only square summable weights (which in itself permits longrange dependence) and i.i.d. innovations. Gorodetskii (1977) improved Davydov's results. 
Taqqu (1975) established (2.16) for $x_{n}(r)=S_{[n r]}$ under a different type of condition on $u_{t}$. He assumed that $u_{t}=G\left(v_{t}\right)$, where $G$ is a possibly nonlinear function and $v_{t}$ is a stationary Gaussian process with zero mean, unit variance and lag- $j$ autocorrelation $\rho(j)=$ $\gamma(j) / \gamma(0) \sim c^{*} j^{2 H-1}$ as $j \rightarrow \infty, 0<c^{*}<\infty, \frac{1}{2}<H<1$. Assuming $E G\left(v_{t}\right)^{2}<\infty$, Taqqu introduced the Hermite rank of $G$ : denoting by $H_{j}(x)=(-1)^{j} e^{\frac{1}{2} x^{2}}\left(d^{j} / d x^{j}\right) e^{-\frac{1}{2} x^{2}}$ the $j$-th Hermite polynomial, and $J(j)=E G\left(v_{t}\right) H_{j}\left(v_{t}\right)$, then the Hermite rank of $G$ is $m=\min _{j \geq 0}(j: J(j) \neq 0)$. Then (2.15) and (2.16) follow when $m=1$; if $m \geq 2$, the series $G\left(v_{t}\right)$ is weakly dependent if $m>1 /(2-2 H)$, otherwise the limiting distribution is nonGaussian. The results of Davydov (1970) and Taqqu (1975) are in fact more general than reported here because they allow for a slowly varying factor in $\gamma(j)$ or $\rho(j)$. Similar results have been given under various other conditions (e.g. Chan and Terrin (1995), Csőrgo and Mielniczuk (1995)).

\section{3. "TYPE II" FRACTIONAL BROWNIAN MOTION}

Levy (1953), Mandelbrot and Van Ness (1968) mention an alternative definition of fractional Brownian motion, as a Holmgren-Riemann-Liouville fractional integral, which we write as

$$
\begin{gathered}
W_{H}(r)=(2 H)^{\frac{1}{2}} \int_{0}^{r}(r-s)^{H-\frac{1}{2}} d B(s), \quad r \geq 0, \\
W_{H}(r)=-(2 H)^{\frac{1}{2}} \int_{r}^{0}(s-r)^{H-\frac{1}{2}} d B(s), \quad r<0 .
\end{gathered}
$$

We call $W_{H}(r)$ "Type II fractional Brownian motion". Clearly $W_{H}(r)$ is again Gaussian with almost surely continuous sample paths, and for $H=\frac{1}{2},(3.1)$ and (3.2) reduce to (2.2) and (2.3), thus nesting $B(r)$ to the same extent as does $B_{H}(r)$. Also we have

$$
E W_{H}(r)=0, \quad E W_{H}^{2}(r)=|r|^{2 H}, \quad r \in R
$$

so that the mean and variance of $W_{H}(r)$ are identical to those of $B_{H}(r)$. However, when $0<r_{1}<r_{2}$

$$
E W_{H}\left(r_{1}\right) W_{H}\left(r_{2}\right)=\frac{1}{2}\left(r_{2}^{2 H}+r_{1}^{2 H}-E\left(W_{H}\left(r_{2}\right)-W_{H}\left(r_{1}\right)\right)^{2}\right),
$$


which does not agree with (2.6), because

$$
\begin{aligned}
E\left(W_{H}\left(r_{2}\right)-W_{H}\left(r_{1}\right)\right)^{2}= & 2 H \int_{0}^{r_{1}}\left\{\left(r_{2}-s\right)^{H-1 / 2}-\left(r_{1}-s\right)^{H-1 / 2}\right\}^{2} d s \\
& +2 H \int_{r_{1}}^{r_{2}}\left(r_{2}-s\right)^{2 H-1} d s \\
= & 2 H\left(r_{2}-r_{1}\right)^{2 H}\left(\frac{1}{2 H}+\int_{0}^{r_{1} /\left(r_{2}-r_{1}\right)}\left\{(1+s)^{H-1 / 2}-s^{H-1 / 2}\right\}^{2} d s\right),
\end{aligned}
$$

which is not the same as $(2.7)$.

Thus the increments of $W_{H}(r)$, even at equally-spaced intervals, are nonstationary, unlike those of $B_{H}(r)$, though

$$
E\left(W_{H}\left(r_{2}\right)-W_{H}\left(r_{1}\right)\right)^{2} \sim 2 H A(H)^{2}\left(r_{2}-r_{1}\right)^{2 H} \text { as } \frac{r_{1}}{r_{2}-r_{1}} \rightarrow \infty
$$

and

$$
E\left(W_{H}\left(r_{2}\right)-W_{H}\left(r_{1}\right)\right)^{2} \sim\left(r_{2}-r_{1}\right)^{2 H} \text { as } \frac{r_{1}}{r_{2}-r_{1}} \rightarrow 0,
$$

the latter expression agreeing with (2.7). Consider also the sequence of increments

$$
w_{H}(j)=W_{H}(j+1)-W_{H}(j), j=0,1, \ldots
$$

We have $E w_{H}(j)=0$ and

$$
\operatorname{Var}\left(w_{H}(j)\right)=g(j)
$$

where

$$
g(t)=1+2 H \int_{0}^{t}\left\{(1+s)^{H-\frac{1}{2}}-s^{H-\frac{1}{2}}\right\}^{2} d s .
$$

Since $1 \leq g(t) \leq 2 H A(H)$, the "Type II" increment variance is bounded below by that of "Type I", due to our choice of multiplying constant $(2 H)^{\frac{1}{2}}$ for the former. The autocovariances are

$$
\operatorname{Cov}\left(w_{H}(j), w_{H}(k)\right)=\frac{1}{2}(I+I I+I I I+I V), j>k,
$$

where

$$
\begin{aligned}
I & =-(j-k)^{2 H} g\left(\frac{k+1}{j-k}\right), I I=(j-k+1)^{2 H} g\left(\frac{k}{j-k+1}\right), \\
I I I & =(j-k-1)^{2 H} g\left(\frac{k+1}{j-k-1}\right), I V=-(j-k)^{2 H} g\left(\frac{k}{j-k}\right),
\end{aligned}
$$


and in case $j=k+1, I I I=0$. It is then readily seen that as $\frac{k}{j-k} \rightarrow 0$

$$
\operatorname{Cov}\left(w_{H}(j), w_{H}(k)\right) \sim \frac{1}{2}\left((j-k+1)^{2 H}-2(j-k)^{2 H}+(j-k-1)^{2 H}\right),
$$

which agrees with (2.10); in particular, we have, for $j, k \geq 0$

$$
E w_{H}(0) w_{H}(k)=E b_{H}(j) b_{H}(j+k) .
$$

The greater dependence on the origin in $W_{H}(r)$, relative to $B_{H}(r)$, was offered as a criticism by Mandelbrot and Van Ness (1968). Another drawback with the joint distributions of $W_{H}(r)$ is that the $H-s s$ property only applies insofar as univariate marginal distributions are concerned. The possibility to provide type II fractional Brownian motion with an harmonizable representation in the sense of (2.12) is still an open field for research.

Three reasons can be advanced for interest in $W_{H}(r)$. First, it is defined on the same domain as $B(r)$. Second, while $B_{H}(r)$ is well defined only for $0<H<1$ (the integral $D(H)$ diverges when $H \geq 1), W_{H}(r)$ is defined for all $H>0$. The value of this can be seen in connection with our third point, which indicates how $W_{H}(r)$ can describe the limit behaviour of certain nonstationary fractional processes. Consider a sequence $\eta_{t}$ which has zero mean and is covariance stationary with spectral density $g(\lambda)$ such that

$$
0<g(0)<\infty
$$

Thus $\eta_{t}$ is "short-range dependent", cf. (1.7). Introduce the sequence

$$
\begin{aligned}
\eta_{t}^{*} & =\eta_{t}, \quad t \geq 1 \\
& =0, \quad t \leq 0
\end{aligned}
$$

and define

$$
y_{t}=(1-L)^{-\frac{1}{2}-H} \eta_{t}^{*}, \quad t \geq 1
$$

where $L$ is the lag operator, and

$$
(1-L)^{-\frac{1}{2}-H}=\sum_{j=0}^{\infty} \psi_{j} L^{j}, \quad \psi_{j}=\frac{\Gamma\left(j+H+\frac{1}{2}\right)}{\Gamma\left(H+\frac{1}{2}\right) \Gamma(j-1)},
$$


where $\Gamma$ is the Gamma function. For $H>0, y_{t}$ is a nonstationary process. For example, when $H=\frac{1}{2}, y_{t}$ has a unit root, while for $H=\frac{3}{2}$, it has two unit roots. When $2 H$ is not an odd integer we can call $y_{t}$ a fractionally integrated process with integration order $H+\frac{1}{2}$.

The convergence

$$
\Gamma\left(H+\frac{1}{2}\right)\left\{\frac{H}{\pi n^{2 H-2} g(0)}\right\}^{\frac{1}{2}} y_{[n r]} \Rightarrow W_{H}(r), \quad 0<r \leq 1,
$$

has been considered by Akonom and Gourieroux (1987), Silveira (1991) and Marinucci and Robinson (1997) assuming the $\eta_{t}$ are i.i.d., or a more general linear process with i.i.d. or absolutely regular innovations. The latter reference provides a multivariate generalization of (3.10). Note that for $H=\frac{1}{2} y_{n}=\sum_{t=1}^{n} \eta_{t}$ so (3.10) is equivalent to (1.8) (with $\eta_{t}=u_{t}$ ), and indeed to (1.6) in case the $\eta_{t}$ are i.i.d. . The convergence (3.10), combined with the continuous mapping theorem, is useful in characterizing the limit distribution of various statistics arising in inference on nonstationary, possibly fractionally integrated processes, for example in cointegration analysis of economic time series.

It is of some interest to note that $W_{H}(r)$ is taken for granted as the proper definition of fractional Brownian motion in the bulk of the econometric time series literature, whereas the probabilistic literature focuses on $B_{H}(r)$. This dichotomy mirrors differing definitions of nonstationary fractionally integrated processes. One definition, $y_{t}$, which led to $W_{H}(r)$, is given in (3.8). The other, which led to $B_{H}(r)$, is prompted by (1.4),

$$
x_{t}=u_{1}+\ldots+u_{t}, \quad t \geq 1
$$

where $u_{t}$ satisfies (2.14). Both have integration order $H+\frac{1}{2}$, but (3.10) allows only $H<1$. To compare (3.8) and (3.11) when $0<H<1$, we rewrite (3.8) as a partial sum of innovations, i.e.

$$
y_{t}=u_{1}^{*}+\ldots+u_{t}^{*}, \quad t \geq 1
$$

where

$$
u_{t}^{*}=(1-L) y_{t}=\sum_{j=0}^{t-1} a_{j} \eta_{t-j}
$$

and

$$
a_{j}=\frac{\Gamma\left(j+H-\frac{1}{2}\right)}{\Gamma\left(H-\frac{1}{2}\right) \Gamma(j-1)} \sim c j^{H-\frac{3}{2}} \text { as } j \rightarrow \infty, 0<c<\infty
$$


see Abramowitz and Stegun (1970), formula 6.1.47; because of the truncation in $\eta_{t}^{*}$, $u_{t}^{*}$ is not covariance stationary. Now in case $u_{t}$ and $u_{t}^{*}$ have the same short-range dependent input, so that

$$
u_{t}=\sum_{j=0}^{\infty} a_{j} \eta_{t-j}
$$

(which satisfies (2.14), in view of (3.6) and (3.13)) we infer from (3.12) that

$$
u_{t}-u_{t}^{*}=\sum_{j=t}^{\infty} a_{j} \eta_{t-j}, \quad t \geq 1
$$

In case (3.6) is extended to

$$
0<g(\lambda) \leq C<\infty, \quad \pi<\lambda \leq \pi,
$$

the deviation (3.14) has variance

$$
\begin{aligned}
\int_{-\pi}^{\pi}\left|\sum_{j=t}^{\infty} a_{j} e^{i j \lambda}\right|^{2} g(\lambda) d \lambda & \leq 2 \pi C \sum_{j=t}^{\infty} a_{j}^{2} \\
& =O\left(t^{2 H-2}\right) \rightarrow 0
\end{aligned}
$$

as $t \rightarrow \infty$, because $a_{j}=O\left(j^{H-3 / 2}\right)$.

From (2.4) and (3.1) we may write down an identity between $B_{H}(r)$ and $W_{H}(r)$ for $r \geq 0$ :

$$
\begin{aligned}
B_{H}(r) & =\frac{1}{A(H)}\left\{\frac{1}{(2 H)^{\frac{1}{2}}} W_{H}(r)+I_{H}(r)\right\} \\
& =\{1+2 H D(H)\}^{-\frac{1}{2}} W_{H}(r)+A(H)^{-1} I_{H}(r),
\end{aligned}
$$

where

$$
I_{H}(r)=\int_{-\infty}^{0}\left\{(r-s)^{H-\frac{1}{2}}-(-s)^{H-\frac{1}{2}}\right\} d B(s)
$$

Thus $B_{H}(r)$ is composed of two independent components, one of them a scaled $W_{H}(r)$.

Occasionally the different definitions of fractional Brownian motion on the one hand, and of fractionally integrated time series on the other, have led the definitions (3.11) and $(2.14) /(2.15)$ of fractionally integrated $x_{t}$ to be incorrectly associated with $W_{H}(r)$. An important early theoretical econometric contribution to the literature is Sowell (1990). This author considered the limiting distribution of the least-squares estimate of the coefficient 
of a first-order autoregression in case the true coefficient is actually 1 and the innovations have long-range or negative dependence. Sowell asserted under conditions assuming (2.14) and (2.15) with $x_{n}(r)=S_{[n r]}$ given by (1.3) and (1.4), or equivalently $x_{n}(r)=x_{[n r]}$ given by (3.11), that

$$
c^{-\frac{1}{2}} n^{-H} x_{n}(r) \Rightarrow W_{H}(r), \quad 0<r \leq 1, \quad 0<H<1,
$$

in contradiction to (2.16). Consequently, Sowell's Theorem 3 requires correction by simply replacing $W_{H}$ by $B_{H}$. To be precise, if $\widehat{\beta}$ is the least-squares estimate of $x_{t}$ on $x_{t-1}$, $t=2, \ldots, n$, then under (3.11) and regularity conditions implying (2.13)

$$
\begin{gathered}
n(\widehat{\beta}-1) \Rightarrow \frac{\frac{1}{2} B_{H}^{2}(1)}{\int_{0}^{1} B_{H}^{2}(r) d r}, \quad \frac{1}{2}<H<1, \\
n^{2 H}(\widehat{\beta}-1) \Rightarrow-\frac{H \Gamma\left(\frac{1}{2}+H\right) / \Gamma\left(\frac{3}{2}-H\right)}{\int_{0}^{1} B_{H}^{2}(r) d r}, \quad 0<H<\frac{1}{2} .
\end{gathered}
$$

Related work of Dolado and Marmol (1996) and Cappuccio and Lubian (1997) also appears to make use of (3.15) rather than (2.16) and can be corrected in a similar way. On the other hand, also in a related context, Chan and Terrin (1995) and Jeganathan (1996) make appropriate use of (2.16).

\section{CONCLUSIONS}

This paper has discussed two alternative definitions of nonstationary fractional processes which have arisen in the literature, the first prompted by (3.11), the other given in (3.8). It was pointed out that associated functional central limit theorems lead to different types of fractional integrals, which we labelled "Type I" and "Type II" fractional Brownian motion. This distinction has sometimes been overlooked in the econometric literature, leading to the definition (3.11) being incorrectly associated with "Type II", rather than "Type I", fractional Brownian motion, and we indicated the implications for some published results. Properties of "Type I" and "Type II" fractional Brownian motion have been compared. 


\section{REFERENCES}

Abramowitz, M. and Stegun, I. (1970) Handbook of Mathematical Functions, Dover, New York

Akonom, J. and Gourieroux, C. (1987) "A Functional Central Limit Theorem for Fractional Processes", Technical Report \#8801, CEPREMAP, Paris.

Billingsley, P. (1968) Convergence of Probability Measures, Wiley, New York

Brown, B.M. (1971) "Martingale Central Limit Theorems", Annals of Mathematical Statistics, 42, 59-66

Cappuccio, N. and Lubian, D. (1997) "Spurious Regression between $I(1)$ Processes with Long Memory Errors", Journal of Time Series Analysis, 18, 341-354

Chan, N.H. and Terrin, N. (1995) "Inference for Unstable Long-Memory Processes with Applications to Fractional Unit Autoregressions", Annals of Statistics, 23, 1662-1683

Csőrgo, S. and Mielniczuk, J. (1995) "Distant Long-Range Dependent Sums and Regression Estimation", Stochastic Processes and their Applications, 59, 143-155

Davydov, Y. (1970) "The Invariance Principle for Stationary Processes", Theory of Probability and its Applications, 15, 487-498

Dobrushin, R.L. (1979) "Gaussian and their Subordinated Self-Similar Random Generalized Fieds", Annals of Probability, 7, 1-28

Dobrushin, R.L. and Major, P. (1979) "Non-Central Limit Theorems for Non-Linear Functions of Gaussian Fields", Zeitschrift fur Wahrscheinlichkeitstheorie und verwandte Gebiete, 50, 27-52

Dolado, J.J. and Marmol, F. (1996) "Efficient Estimation of Cointegrating Relationships among Higher Order and Fractionally Integrated Processes", Banco de EspanaServicio de Estudios, Documento de Trabajo 9617

Donsker, M.D. (1951) "An Invariance Principle for Certain Probability Limit Theorems", Memoirs of the American Mathematical Society, 6-12

Gorodetskii, V.V. (1977) "On Convergence to Semi-Stable Gaussian Processes", The- 
ory of Probability and its Applications, 22, 498-508

Hannan, E.J. (1979) "The Central Limit Theorem in Time Series Regression", Stochastic Processes and their Applications, 9, 281-289

Herrndorf, N. (1984) "A Functional Central Limit Theorem for Weakly Dependent Sequences of Random Variables", Annals of Probability, 12, 141-153

Heyde, S.C. (1981) "Invariance Principles in Statistics", Review of the International Statistical Institute, 49, 143-151

Jeganathan, P. (1996) "On Asymptotic Inference in Cointegrated Time Series with Fractionally Integrated Errors", Department of Statistics, University of Michigan, Technical Report \#277

Levy, P. (1953) "Random Functions: General Theory with Special Reference in Laplacian Random Functions", University of California Publications in Statistics, 1, 331-390

Mandelbrot, B.B. and Van Ness, J.W. (1968) "Fractional Brownian Motions, Fractional Noises and Applications", SIAM Review, 10, 422-437

Marinucci, D. and Robinson, P.M. (1998) "Weak Convergence for Multivariate Fractional Processes", in preparation

McLeish, D.L. (1977) "On the Invariance Principle for Nonstationary Martingales", Annals of Probability, 5, 616-621

Phillips, P.C.B and Durlauf, N. (1986) "Multiple Time Series Regression with Integrated Processes", Review of Economic Studies 53, 475-495

Phillips, P.C.B. and Solo, V. (1992) "Asymptotics for Linear Processes", Annals of Statistics, 20, 971-1001

Prohorov, Y.V. (1956) "Convergence of Random Processes and Limit Theorems in Probability Theory", Theory of Probability and its Applications, 1, 157-213

Samorodnitsky, G. and Taqqu, M.S. (1994) Stable Non-Gaussian Random Processes, Chapman and Hall, New York.

Silveira, G. (1991) Contributions to Strong Approximations in Time Series with Applications in Nonparametric Statistics and Functional Central Limit Theorems, Ph.D. thesis, University of London 
Skorohod, A.V. (1956) "Limit Theorems for Stochastic Processes", Theory of Probability and its Applications, 1, 261-290

Sowell, F.B. (1990) "The Fractional Unit Root Distribution", Econometrica, 58, 495505

Taqqu, M.S. (1975) "Weak Convergence to Fractional Brownian Motion and to the Rosenblatt Process", Zeitschrift fur Wahrscheinlichkeitstheorie und verwandte Gebiete, 31, 287-302

Taqqu, M.S. (1979) "Convergence of Integrated Processes of Arbitrary Hermite Rank", Zeitschrift fur Wahrscheinlichkeitstheorie und verwandte Gebiete, 50, 53-83

Verwaat, W. (1985) "Sample Paths of Self-Similar Processes with Stationary Increments", Annals of Probability, 13, 1-27

Weyl, H. (1917) "Bemerkungen zum Begriff der Differential-Quotenten gebrochener Ordnung", Vierteljschr. Naturforsch. Ges. Zurich, 62, 296-302

Wooldridge, J.M. and White, H. (1988) "Some Invariance Principles and Central Limit Theorems for Dependent Heterogenous Processes", Econometric Theory 4, 210-230

Yong, C.H. (1974) Asymptotic Behaviour of Trigonometric Series, Chinese University of Hong Kong, Hong Kong

Zygmund, A. (1977) Trigonometric Series, 2nd edition, Cambridge University Press, Cambridge 